\title{
Safety and Efficacy of Bedside Peritoneal Dialysis Catheter Placement in the COVID-19 Era: Initial Experience at a New York City Hospital
}

\author{
Mariana Vigiola Cruz $^{1}$ (D) $\cdot$ Omar Bellorin ${ }^{1} \cdot$ Vesh Srivatana $^{2} \cdot$ Cheguevara Afaneh $^{1}$
}

Published online: 26 May 2020

(C) Société Internationale de Chirurgie 2020

\begin{abstract}
Introduction Acute kidney injury (AKI) requiring renal replacement therapy (RRT) is common in critically ill patients with COVID-19. Unparalleled numbers of patients with AKI and shortage of dialysis machines and operative resources prompted consideration of expanded use of urgent-start peritoneal dialysis (PD) and evaluation of the safety and efficacy of bedside surgical placement of PD catheters.

Study design Bedside, open PD catheter insertions were performed in early April 2020, at a large academic center in New York City. Patients with SARS-CoV-2 infection and AKI and ambulatory patients with chronic kidney disease and impending need for RRT were included. Detailed surgical technique is described.

Results Fourteen catheters were placed at the bedside over 2 weeks, 11 in critically ill COVID-19 patients and three in ambulatory patients. Mean patient age was 61.9 years (43-83), and mean body mass index was 27.1 (20-37.6); four patients had prior abdominal surgery. All catheters were placed successfully without routine radiographic studies or intraoperative complications. One patient (7\%) experienced primary nonfunction of the catheter requiring HD. One patient had limited intraperitoneal bleeding while anticoagulated, which was managed by mechanical compression of the abdominal wall and temporarily holding anticoagulation. All other catheters had an adequate function at 3-18 days of follow-up.

Conclusions Bedside placement of PD catheters is safe and effective in ICU and outpatient clinic settings. Our surgical protocols allowed for optimization of critical hospital resources, minimization of hazardous exposure to healthcare providers and a broader application of urgent-start PD in selected patients. Long-term follow-up is warranted.
\end{abstract}

Mariana Vigiola Cruz

mav9206@med.cornell.edu

1 Department of Surgery, New York Presbyterian HospitalWeill Cornell Medicine, 525 East 68th Street Box 294, New York, NY 10065, USA

2 Department of Nephrology and Hypertension, New York Presbyterian Hospital-Weill Cornell Medicine, New York, NY, USA

\section{Introduction}

Kidney involvement in patients with SARS-CoV-2 infection has been widely reported in the recent literature and experienced firsthand at our institution in the early phase of the COVID-19 pandemic. Some degree of renal dysfunction has been described in nearly two-thirds of hospitalized patients in published series [1], and the progression to renal replacement therapy (RRT) is relatively common. The pathophysiology of acute kidney injury (AKI) and acute renal failure (ARF) as part of COVID-19 is complex, multifactorial and incompletely understood. Emergent 
evidence suggests the possibility of a direct cytopathic effect of the virus itself, along with pro-inflammatory injury secondary to cytokine release, insults related to hypoperfusion and other systemic events [1-3]. The need for dialysis typically occurs during the second week of hospitalization with recent data estimating an incidence of $15 \%$ in critically ill COVID-19 patients [2, 4]. This is a substantial increase from the baseline of approximately $5 \%$ in intensive care unit (ICU) patients previously estimated worldwide [5].

Considering the acute escalation in the proportion of critically ill patients requiring RRT and the rapid expansion of ICU capabilities to over $200 \%$ within our institution even in the early days of the pandemic reaching New York City, workforce issues and shortages in $\mathrm{HD} / \mathrm{CVVH}$ machines and related supplies were quickly predicted [6]. This overwhelming need for urgent renal replacement resources mandated exploration of alternative methodologies. Peritoneal dialysis (PD) has been long investigated and well documented to be appropriate for treatment for acute kidney injury in critically ill populations [7-11]. PD offers several potential advantages over intermittent hemodialysis (HD) or variations of continuous renal replacement therapy (CRRT) by being a simple, gentle and efficient RRT method, generally beneficial in patients with hemodynamic instability, in patients in whom systemic anticoagulation is contraindicated [7] and in low-resource healthcare settings [8-10]. Furthermore, a hypercoagulable state is now known to be associated with COVID-19 [12, 13], causing not only venous thromboses, but also recurrent thrombosis of the dialysis circuits despite systemic anticoagulation in our patients. This often resulted in substantial loss of blood volume and undesired interruptions in therapy in some patients initially undergoing HD/ CRRT, making independence of vascular access for dialysis specifically advantageous in this critically ill population.

There are various established modalities of insertion of PD catheters, including both percutaneous and surgical approaches [14]. Laparoscopy-assisted placement is standard of care in our surgical practice and broadly in the USA, with catheter insertion by open surgical dissection being less commonly performed today. The ongoing healthcare crisis presented several limitations to laparoscopic placement, including inaccessibility of operating rooms now converted to COVID-19 ICUs, unavailability of Anesthesiology teams and nurses now covering these units and shortages of personal protective equipment (PPE). Additionally, initial reports questioned the safety of performing laparoscopy in patients with SARS-CoV-2 infection [15-17], making an open approach a more desirable technique. Even if operating rooms and staff had been available, however, for both technical reasons in such critically ill patients and for prevention of viral spread outside of negative-pressure rooms, moving inpatients to an operating room setting would not have been feasible. For these reasons, our team was tasked with creating a protocol for open placement of PD catheters at the bedside in selected patients requiring urgent dialysis. We aim to share our initial experience with bedside placement in both ICU patients with COVID-19-related AKI and in ambulatory patients with chronic kidney disease (CKD) and impending need for RRT. We felt that beyond creating access in the critically ill inpatients, it was important to demonstrate the ability to extrapolate this technique to the ambulatory setting under local anesthetic only, as these outpatients would otherwise have been unable to have PD catheters placed in a timely fashion due to mandated OR restrictions for emergency cases exclusively.

\section{Materials and methods}

Our initial experience spans the first two and half weeks of April 2020 at a large academic institution in New York City. Patients were referred to the surgical team by nephrologists evaluating AKI/ARF in the COVID-19 ICUs or following as outpatients for CKD. Decisions on recommendation for PD in inpatients were made based on system-based considerations, particularly shortages of CRRT machines and dialysate, as well as individual factors, such as persistent hypotension not allowing intermittent HD, and repeated thrombosis of dialysis lines or circuits. Outpatients were selected based on the patient's desire and ability to pursue and manage PD, and their adequacy as surgical candidates. Once seen and examined by the surgeons, patients that had limited or no prior abdominal surgery were considered for bedside PD catheter placement. Patients with extensive or poorly documented surgical histories were maintained on HD/CRRT when possible or had laparoscopic insertion of the PD catheters and are not included in this report. Outpatient telemedicine surgical consultations for patients with chronic kidney disease imminently requiring dialysis yielded several suitable candidates for bedside placement of PD catheters in surgical clinic.

\section{Technique}

Following informed consent, the ICU patient is positioned on the bed supine with the arms to the sides. Therapeutic anticoagulation is held, and antibiotics are administered per usual preoperative protocols.. A monopolar electrocautery system is brought to the bedside. To minimize utilization of unnecessary resources and streamline our setup, a list of instruments (Table 1) was provided to the Central Sterile 
Department and surgical trays specific to this procedure were prepared. A Mayo stand or side table is set up with the necessary sterile supplies including a sterile handheld light source. The surgeons don appropriate PPE, including N95 respirators, eye goggles, face shields, head and shoe coverings, and sterile gowns and double gloves prior to entering the patient space with all verified equipment. The patient is typically sedated per ICU management and administered additional doses of intravenous analgesic or sedatives as needed, with the addition of local anesthesia in select cases.

Once the patient is prepped and draped, a $3-\mathrm{cm}$ incision is made in the right upper quadrant, approximately $2-3 \mathrm{~cm}$ lateral to the midline (Fig. 1). The subcutaneous tissue is dissected down to the anterior rectus sheath using monopolar energy and blunt retraction using handheld retractors appropriate for the patient's habitus. A Weitlaner retractor is then placed to maintain exposure, and two Allis clamps are placed side-by-side on the anterior rectus sheath. The sheath is then sharply incised, and the muscle fibers are bluntly dissected laterally to expose the posterior rectus sheath. The Allis clamps are then transferred to the posterior rectus sheath, and this is pulled anteriorly. To avoid inadvertently grabbing bowel, the Allis clamps are then sequentially unclamped and re-clamped. The posterior rectus sheath and peritoneum are then sharply incised. A 0 -Vicryl purse-string suture on a GU- or UR-style needle is placed but not tied around the posterior fasciotomy. The dilator/peel-away sheath combination is advanced toward the pelvis, and the dilator is then withdrawn. A right-sided $62.5 \mathrm{~cm}$ swan neck curl, double-cuffed peritoneal dialysis

Table 1 Contents of sterile surgical tray. List of instruments to the Central Sterile Department to prepare specific surgical trays

Contents of sterile surgical tray

Halsted/hemostat forceps $\times 4$

Schnidt tonsil forceps $\times 2$

Kelly forceps $\times 1$

Allis tissue forceps $\times 2$

DeBakey forceps $\times 2$

Rat tooth issue forceps $\times 1$

Adson forceps $\times 1$

Towel clamp $\times 4$

Mayo Hegar needle holder $\times 1$

Metzenbaum scissor

Mayo scissor

Small Richardson retractor $\times 2$

Army-Navy retractor $\times 2$

Blunt Weitlaner retractor $\times 2$

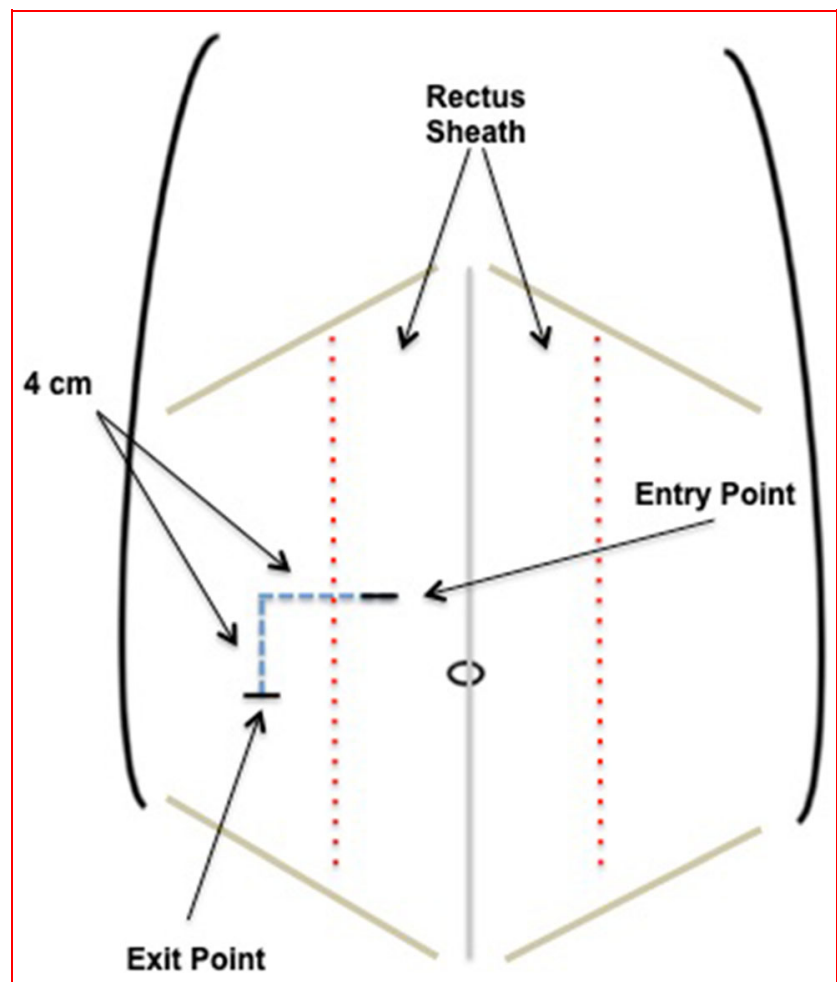

Fig. 1 Anatomic landmarks. Catheter entry site incision in the right upper quadrant, approximately $2-3 \mathrm{~cm}$ lateral to the midline and tunneled toward the exit site at $4 \mathrm{~cm}$ inferior and $4 \mathrm{~cm}$ lateral to the introduction site

catheter is then introduced through the sheath after soaking it in heparinized saline (5000 units in $500 \mathrm{cc}$ ). The sheath is then peeled away as the dialysis catheter is further introduced so the inner cuff is at the level of the posterior rectus sheath, ensuring the anterior marking line on the catheter remains in the proper orientation. The catheter is then secured to the posterior sheath by incorporating a pass of the previously placed 0 -Vicryl purse-string suture through the soft material of the inner cuff, taking care not to violate the catheter tubing. Once tied, this should create a tight seal around the catheter and allow for immediate initiation of PD. The anterior fascia is then partially closed around the catheter with a second 0-Vicryl suture, confirming no kinks are caused in the tubing.

Tunneling the catheter and outer cuff is performed by measuring the exit site at $4 \mathrm{~cm}$ inferior and $4 \mathrm{~cm}$ lateral to the catheter introduction site. A punctate incision is made on the skin, and a subcutaneous tunnel is created by introducing a small pointed clamp through the skin exit site and into the open incision, and the catheter is grasped and pulled through. The outer cuff should land $1-2 \mathrm{~cm}$ proximal to the exit site within the subcutaneous tissues. The catheter should be flushed with heparinized saline following each critical step of the procedure to ensure adequate 
flow. The catheter tip is then accessorized with the appropriate locking adapter, PD transfer set and sterile catheter cap. The open incision is then closed in two layers with absorbable suture, and a sterile dressing is placed. A chlorhexidine-gel containing dressing is placed at the catheter exit site.

Insertion of the catheters in surgical clinic for ambulatory patients with CKD mimics the ICU bedside technique, with slight modifications. Outpatients are prescribed a firstgeneration cephalosporin pre-procedure and for $24 \mathrm{~h}$ postprocedure. The patient is prescribed an oxycodone/APAP dose to take prior to arrival in clinic. Local anesthetic is infiltrated into the skin and soft tissue as needed. With proper administration of local anesthetic, no systemic agents are required for sedation.

\section{Guidelines for addressing common surgical issues post-procedure}

\section{Leakage}

Peri-catheter leakage of dialysate fluid may occur secondary to poor sealing of the posterior sheath or herniation around the tubing. This can be best avoided by following appropriate technique during placement and addressed in the postoperative setting with pressure-type dressing overlying the entry site and placement of an abdominal binder. If feasible, lower dwell volumes can be considered.

\section{Flow dysfunction}

Flow dysfunction generally represents a mechanical problem. Inflow malfunction or difficulty with flushing of the catheter can be initially addressed by power flushes with heparinized saline. If this fails, reexploration may be warranted to salvage the catheter, ensuring no kinking occurs in the tunneling or the fascial closures. If there is no obstruction along the abdominal wall, clogging of the catheter by debris or clots, or obstruction of the tip by bowel or omentum should be suspected and the catheter should be withdrawn and reintroduced at a different angle or repositioned more laterally.

Outflow dysfunction is generally signaled by failure to drain at an adequate rate. The greatest risk factors for this are adhesions or obesity, especially morbid obesity. First, aggressively flushing the catheter with $500 \mathrm{cc}$ of heparinized saline and rotating the patient to the decubitus positions should be attempted, as this may allow the catheter to flip or move to a more favorable location. If this fails, an aggressive bowel regimen should be initiated to ensure no obstruction by hard stool is occurring. If necessary, another form of renal replacement therapy should be initiated and the above maneuvers reattempted in 24-48 h.

\section{Results}

In the initial series of bedside insertions, 14 catheters were placed by one of two attending surgeons and a surgical fellow over a 2-week period. Eleven patients had COVID19 confirmed by testing and were critically ill and intubated in ICU settings. Three ambulatory patients were asymptomatic and were not tested for SARS-CoV-2 infections. Analysis of demographic data (Table 2) included mean age of 61.9 years (range 43-83 years) and body mass index (BMI) mean of $27.1 \mathrm{~kg} / \mathrm{m}^{2}$ (range 20-37.6). Two patients were female and four had had prior abdominal surgery, including cesarean section, appendectomy and ventral and inguinal hernia repair. Review of medical history demonstrated six patients with CKD, four patients with type 2 diabetes mellitus, nine patients with hypertension, six patients with significant cardiac history including coronary artery or valvular disease or congestive heart failure, three patients with chronic respiratory disease including asthma and chronic obstructive pulmonary disease and two patients with immune-related conditions, one with history of lymphoma and prior stem cell transplant and other with rheumatoid arthritis on chronic methotrexate.

At the time of insertion, all ICU inpatients were critically ill and intubated due to COVID-19-related respiratory failure, four patients with fraction of inspired oxygen requirement $>60 \%$ or positive end-expiratory pressure $>10 \mathrm{cmH} 20$ and five patients requiring vasopressor support. One patient was requiring nightly prone positioning, and PD was adjusted to occur during her supine periods. Seven patients were already undergoing HD/ CRRT. Mean length of hospital admission on day of procedure was 10.9 days (range 2-28). Ten of these patients were therapeutically anticoagulated before and after the procedure; the remaining one having anticoagulation was held due to recent bleeding concerns. One of the outpatients had a history of atrial fibrillation and was chronically anticoagulated with warfarin, which was held for 5 days pre-procedure.

All catheters were placed successfully and without unintended or unforeseen intraoperative events. All patients tolerated the procedure well with sedation and analgesics/local anesthetic as described above; two patients had paralytic agents ongoing prior to procedure. Insertion times improved rapidly with a steep learning curve in the setup and optimization of technical details, with operative times averaging approximately $40 \mathrm{~min}$ and ranging between 25 and $65 \mathrm{~min}$. No routine radiographic studies were used to verify catheter tip location post-procedure.

PD was initiated within $24 \mathrm{~h}$ of placement in all ICU patients and within 7 days in the ambulatory patients. Adequacy of catheter function was assessed by 
2468

World J Surg (2020) 44:2464-2470

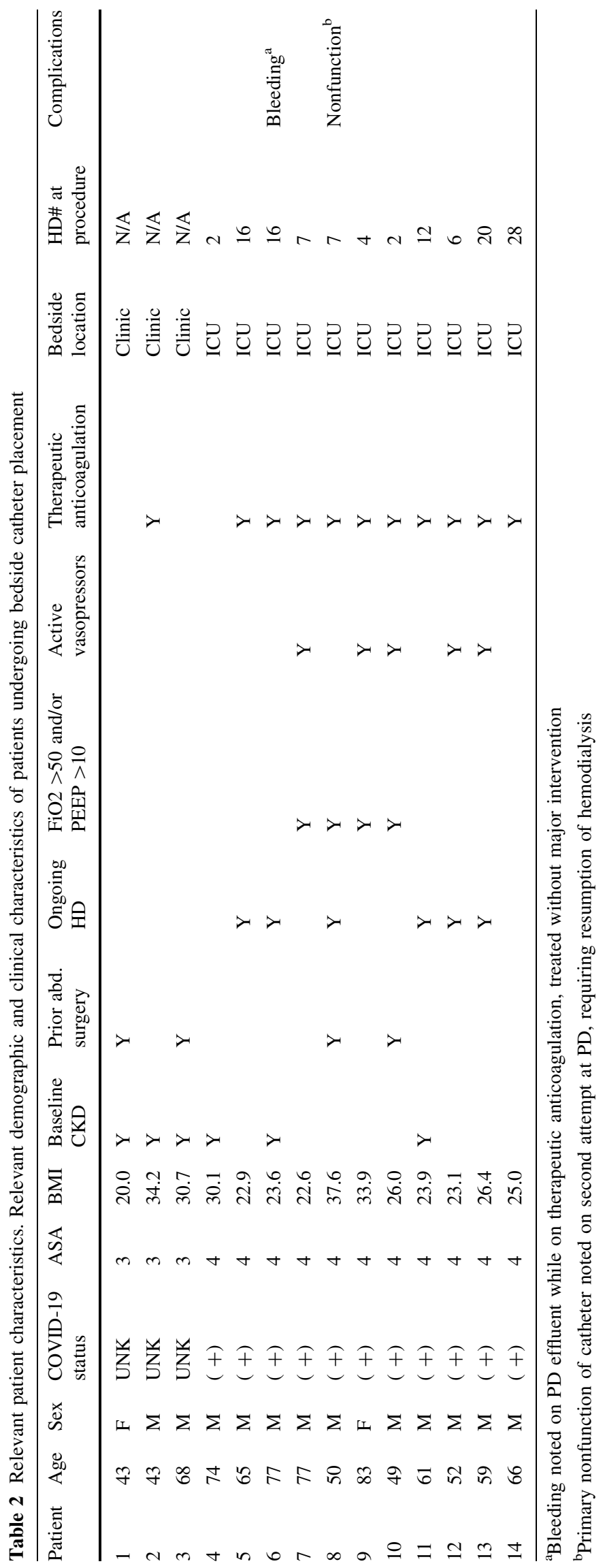

Springer 
chart review and direct communication with the ICU and nephrology teams. The quality of dialysis was evaluated daily by the nephrology team and was, on average, calculated to be equivalent to $\geq 3$ days of hemodialysis per week for all patients, which is considered physiologically adequate.

One patient had primary nonfunction of the catheter (7\%), with failure of outflow at second attempt at PD and persistent dysfunction despite numerous flushes, positional maneuvers and radiographic confirmation of the catheter in the pelvis. This patient had the highest BMI of our series $\left(37.6 \mathrm{~kg} / \mathrm{m}^{2}\right)$ and had history of open appendectomy. Another patient had sanguineous dialysate output on postprocedure day 3 with an associated $1 \mathrm{~g} / \mathrm{dL}$ drop in serum hemoglobin over $36 \mathrm{~h}$ while therapeutically anticoagulated. An abdominal binder was placed, and heparin infusion was paused for $24 \mathrm{~h}$. Return to clear dialysate output was noted without further intervention. Two other patients had abdominal films for independent reasons and incidentally demonstrated the catheter tips adequately located in the pelvis. Follow-up reported here ranged between 3-18 days after the procedure. Two ICU patients were extubated and were able to leave the ICU. One patient expired due to complications of COVID-19 during our short follow-up period; the calculated dialysis parameters demonstrated his PD was equivalent to $>3$ times per week of HD.

\section{Discussion}

In our limited series, bedside placement of peritoneal dialysis catheters in the early phases of the COVID-19 pandemic offered a safe and effective option for establishment of access for renal replacement therapy. Our protocol for open insertion was found to be feasible, efficient and reproducible, with a primary nonfunction rate of $7 \%$ despite the lack of radiographic guidance, which is within the recommended rate of $<20 \%$ [18-20]. In offering this procedure in a reliable and timely fashion, the surgical team promoted institutional efforts to optimize critical personnel and material resources, namely in the allocation of dialysis machines and supplies, operating room space and protective equipment. Considering national shortages, it was important that only two sets of PPE were required for each catheter insertion, far less than the number that would have been required to protect a fully staffed operating room. Additionally, bedside placement of the catheters by a surgeon and an assistant alone limited the hazardous exposure of additional healthcare professionals, including anesthesia providers, nurses and surgical and radiologic technologists.

The development of urgent-start PD programs has been a goal of hospital quality improvement initiatives in recent years [21, 22]. A commonly perceived limitation to this is the ability to readily obtain intraperitoneal catheter placement. In our series, for patients with ARF not yet undergoing hemodialysis, PD catheters were generally placed within $24 \mathrm{~h}$ of consultation by the Nephrology team. In more than one instance, the catheter insertion procedure was completed within $2 \mathrm{~h}$ of the surgeons receiving initial consultation for urgent-start PD. We demonstrated that an experienced surgical team with efficient protocols and reproducible methods at the bedside can match the promptness and safety of a routine central venous catheter insertion for initiation of urgent hemodialysis.

Randomized studies and meta-analyses have found the outcomes of PD in critically ill patients with AKI to be at least equivalent to HD/CRRT alternatives [7, 8, 10]. Some studies have reported higher rates of survival, lower rates of infectious complications, improved recovery of kidney function, lower cost and better quality of life scores in patients treated with PD [7, 10, 11]. Additionally, research suggests that PD, compared to HD, may achieve significant cost savings if applied to large numbers of patients $[9,10]$. This all will perhaps encourage establishment of urgent-start peritoneal dialysis programs at more institutions in the future.

In nonurgent settings following PD catheter placement, patients undergo a 2-week break-in period where only small volumes of dialysate are infused, in expectation that this diminishes the risk of peri-catheter leaks and that it allows gradual training of patients starting PD at home. Published literature has failed to demonstrate those early or urgent-start dialysis precipitates leaks, and the need for this recommended break-in period is now considered to be mostly theoretical [21, 23].

The ripple effects of this acute wave of renal replacement will have long-lasting effects on the healthcare system. In large-scale studies in the general population, most patients that experience ARF while critically ill are dialysis-independent at hospital discharge [5], but these outcomes are not yet known following COVID-19. With hundreds of patients potentially recovering from severe SARS-CoV-2 infection and even a small proportion requiring RRT post-discharge, the burden of this patient population on outpatient dialysis sites and rehabilitation facilities should be considered. Broadening the applications of PD during initial hospitalization could off-load some of this burden.

Since January of 2019, legislation expanded telehealth services for beneficiaries requiring maintenance dialysis therapy, eliminating geographic restrictions for home dialysis patients [24]. With an immediate need to accommodate social distancing recommendations in the recent weeks, referrals for preoperative telemedicine visits were made for the ambulatory patients included in this series. We have now seen that both initial visit and follow-up can be safely achieved using telemedicine in a variety of 
patients. With further expansions in telemedicine capabilities due to the pandemic, it will likely be feasible for many more discharged patients to continue close monitoring from home for their PD. This will further the efforts in maintaining safety of ambulatory dialysis with less crowding at dialysis facilities, as well as minimizing risk of viral transmission in this vulnerable population [25, 26].

Limitations of our series primarily relate to the small number of patients included and the very short follow-up period. Long-term outcomes must be carefully monitored. Nevertheless, our diverse and critically ill patient population demonstrates the safety of this procedure including in the use of patients requiring prone positioning to optimize oxygenation. Being at the forefront of the COVID-19 pandemic in the USA, however, we were quickly faced with a paradigm shift in surgical practice and a wish to participate in early information sharing to potentially benefit patients across other institutions.

While the current public health crisis has forced upon us an unprecedented challenge, it will also provide exceptional opportunities to learn and adjust to a different era in patient care. Lastly, we hope this fundamental change will be positively reflected in the future practice of American medicine, compelling providers to meticulously consider resource utilization and healthcare spending not only when the means are scarce.

Funding This research did not receive any specific grant from funding agencies in the public, commercial or not-for-profit sectors.

\section{References}

1. Naicker S, Yang CW, Hwang SJ et al (2020) The Novel Coronavirus 2019 epidemic and kidneys. Kidney Int 97(5):824-828

2. Durvasula R, Wellington T, McNamara E, Watnick S (2020) COVID-19 and kidney failure in the acute care setting: our experience from Seattle. Am J Kidney Dis S02726386(20)30618-1

3. Ronco C, Reis T (2020) Kidney involvement in COVID-19 and rationale for extracorporeal therapies. Nat Rev Nephrol 16(6):308-310

4. American Society of Nephrology (2020) Recommendations on the care of hospitalized patients with Covid-19 and kidney failure requiring renal replacement therapy. Accessed 15 Apr 2020

5. Uchino S, Kellum JA, Bellomo R et al (2005) Beginning and ending supportive therapy for the kidney (BEST kidney) investigators. Acute renal failure in critically ill patients: a multinational, multicenter study. JAMA 294:813-818

6. Burgner A, Ikizler TA, Dwyer JP (2020) COVID-19 and the inpatient dialysis unit: managing resources during contingency planning pre-crisis. Clin J Am Soc Nephrol 15(5):720-722

7. Al-Hwiesh A, Abdul-Rahman I, Finkelstein F et al (2018) Acute kidney injury in critically Ill patients: a prospective randomized study of tidal peritoneal dialysis versus continuous renal replacement therapy. Ther Apher Dial 22(4):371-379
8. Chionh CY, Soni SS, Finkelstein FO et al (2013) Use of peritoneal dialysis in AKI: a systematic review. Clin J Am Soc Nephrol 8(10):1649-1660

9. Dias DB, Mendes ML, Caramori JT et al (2020) Urgent-start dialysis: comparison of complications and outcomes between peritoneal dialysis and haemodialysis. Perit Dial Int 896860820915021

10. Liu FX, Gao X, Inglese G et al (2015) A global overview of the impact of peritoneal dialysis first or favored policies: an opinion. Perit Dial Int. 35(4):406-20

11. Liu FX, Ghaffari A, Dhatt $\mathrm{H}$ et al (2014) Economic evaluation of urgent-start peritoneal dialysis versus urgent-start hemodialysis in the United States. Medicine (Baltimore) 93(28):e293

12. Tan CW, Low JGH, Wong WH et al (2020) Critically Ill COVID19 infected patients exhibit increased clot waveform analysis parameters consistent with hypercoagulability. Am J Hematol. Online ahead of print.

13. Terpos E, Ntanasis-Stathopoulos I, Elalamy I et al (2020) Hematological findings and complications of COVID-19. Am J Hematol. Online ahead of print.

14. Sun ML, Zhang Y, Wang B et al (2020) Randomized controlled trials for comparison of laparoscopic versus conventional open catheter placement in peritoneal dialysis patients: a meta-analysis. BMC Nephrol 21(1):60

15. Vigneswaran Y, Prachand VN, Posner MC et al (2020) What is the appropriate use of laparoscopy over open procedures in the current COVID-19 climate? J Gastrointest Surg1-6

16. Di Saverio S, Khan M, Pata F et al (2020) Laparoscopy at all costs? Not now during COVID-19 and not for acute care surgery and emergency colorectal surgery: a practical algorithm from a Hub Tertiary teaching hospital in Northern Lombardy, Italy. J Trauma Acute Care Surg. Online ahead of print.

17. Alp E, Bijl D, Bleichrodt RP et al (2006) Surgical smoke and infection control. J Hosp Infect 62(1):1-5

18. McCormick BB, Bargman JM (2007) Noninfectious complications of peritoneal dialysis: implications for patient and technique survival. J Am Soc Nephrol 18:3023-3025

19. Danielsson A (2007) The controversy of placement of peritoneal dialysis catheters. Perit Dial Int 27:153-154

20. George N, Alexander S, David VG et al (2016) Comparison of early mechanical and infective complications in first time blind, bedside, midline percutaneous Tenckhoff Catheter insertion with ultra-short break-in period in diabetics and non-diabetics: setting new standards. Perit Dial Int 36(6):655-661

21. Ghaffari A, Kumar V, Guest S (2013) Infrastructure requirements for an urgent-start peritoneal dialysis program. Perit Dial Int 33(6):611-7

22. Arramreddy R, Zheng S, Saxena AB et al (2014) Urgent-start peritoneal dialysis: a chance for a new beginning. Am J Kidney Dis 63(3):390-395

23. Crabtree JH, Burchette RJ (2009) Effect of prior abdominal surgery, peritonitis, and adhesions on catheter function and longterm outcome on peritoneal dialysis. Am Surg 75(2):140-7

24. Lew SQ, Sikka N (2019) Operationalizing telehealth for home dialysis patients in the United States. Am J Kidney Dis 74(1):95-100

25. Ikizler TA, Kliger AS (2020) Minimizing the risk of COVID-19 among patients on dialysis. Nat Rev Nephrol (6):311-313

26. Watnick S, McNamara E (2020) On the frontline of the COVID19 outbreak: keeping patients on long-term dialysis safe. Clin J Am Soc Nephrol 15(5):710-713

Publisher's Note Springer Nature remains neutral with regard to jurisdictional claims in published maps and institutional affiliations. 